Inter J Nav Archit Oc Engng (2010) 2:75 86

DOI 10.3744/JNAOE.2010.2.2.075

\title{
Analytic study of a new conceptual propulsion device for ships
}

\author{
Roberto Muscia and Giacomo Sciuto \\ Department of Mechanical Engineering, University of Trieste, Trieste, ITALY
}

\begin{abstract}
In this work the possibility of obtaining a rectilinear motion of bodies partially or totally submerged without using propellers is evaluated. The system propulsion is based on a pair of counter rotating masses that generate the thrust. The fluid-body system has been schematized in order to carry out a very simple model. Using this model an evaluation of the body motion along a longitudinal direction was performed. The motion equations of the system were written and integrated. The external forces applied to the body depend on its velocity in relation to the water. These forces were obtained by fluid dynamic simulations. Regarding the mechanical configuration suggested, the results obtained show that a certain displacement of the body along a fixed direction is obtainable.
\end{abstract}

KEY WORDS: Propulsion system; Dynamics; Vibrations; Motion equations; Centrifugal force; CFD computation.

\section{INTRODUCTION}

The utilization of propellers in the ship industry represents a general solution to movement in the aquatic environment, with limited available alternatives. From the invention of the propeller (Ressel, 1827, Ericsson, 1838, and Smith, 1836) up till to nowadays, more profitable technical or economical alternatives have not yet been designed yet. At present only some water-jet propellers (Colombo, 1985a) for propulsion of small or medium sized boats are sometimes used. This means that propulsion based on classical propellers is very well established. In fact, this system of propulsion has been shown to function effectively. For example, propeller efficiency may change from about 0.5 to 0.7 depending on geometric and manufacturing parameters (Colombo, 1985b). However, the shape of this device is not simple. When the propeller diameter is large, to manufacture it becomes difficult and expensive. Furthermore, scheduled maintenance is particularly costly in these devices. Based on such observations, the attempt for evaluating whether there is a practical alternative with respect to the propeller utilization for boats, ships, and/or submarines can be beneficial. First, this alternative must be evaluated, using a physical principle. Second, to determine its viability, the efficiency and cost effectiveness of the new propulsion system must also be assessed. In this study we investigate the first component, i.e. analyzing the possibility of obtaining a significant displacement of the whole system in a certain direction.

Corresponding author: Roberto Muscia

e-mail:muscia@units.it

\section{BASIC PHYSICAL WORKING}

In order to describe the working principle of the new propulsion system let us consider the simple device illustrated in Fig. 1(a). A mass-point $m$ is hinged by a crank of a length equal to $r$. The slide can horizontally translate. The mass rotates around the hinge and its angular position at time $t$ is fixed by the angle $\theta(t)$. The angular velocity $\dot{\theta}$ is constant. Therefore the centrifugal force $F_{C}$ is applied to $m$ along $r$. Note particularly the horizontal component $F_{C}$ $\cos \theta(t)$ of $F_{C}$. This component causes a symmetric oscillation of the system in the range and its final points are represented in Fig. 1(b) by the two hinge-slides shown by a dotted line. So, the system vibrates horizontally around a central position with displacement $y(t)$, velocity $\dot{y}(t)$, and acceleration $\ddot{y}(t)$ [Fig. 1(b)].

Now let us suppose that during the oscillation another force is applied to the hinge-slide. This force horizontally acts and depends on the velocity $\dot{y}(t)$ of the system. In Fig. 2(a) the above-mentioned force is denoted by by $F_{i d}[\dot{y}(t)]$. It is acting in opposition to $\dot{y}(t)$, that is, $F_{i d}[\dot{y}(t)]$ tends to decrease the velocity of the system. Now, let us observe the situation of the system at time $t+\Delta t$. We can choose the value of $\Delta t$ in such a way that at $t+\Delta t$ the hinge-slide has already reached the maximum displacement along the right direction and it is coming back. Then, because we have defined the function $F_{i d}(\dot{y})$ so that it is always opposed to $\dot{y}$, the direction of $F_{i d}[\dot{y}(t+\Delta t)]$ is going to the right. The horizontal resultant of the forces applied to the device at time $t$ is [see Fig. 2(a)].

$$
R(t)=F_{c} \cos \theta(t)-F_{i d}[\dot{y}(t)]
$$




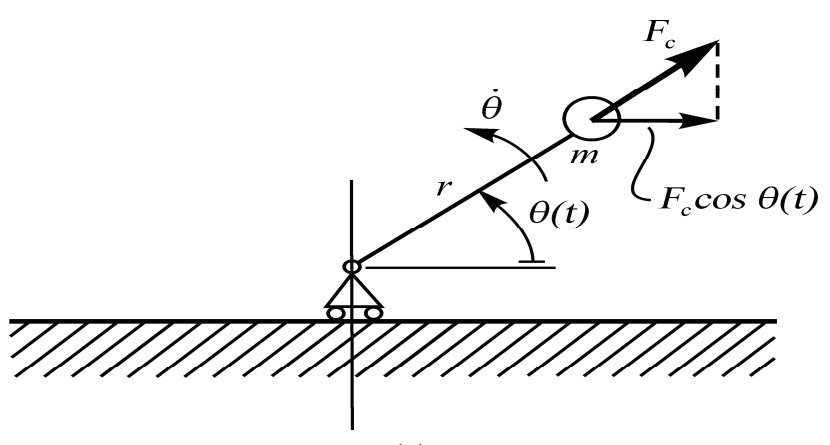

(a)

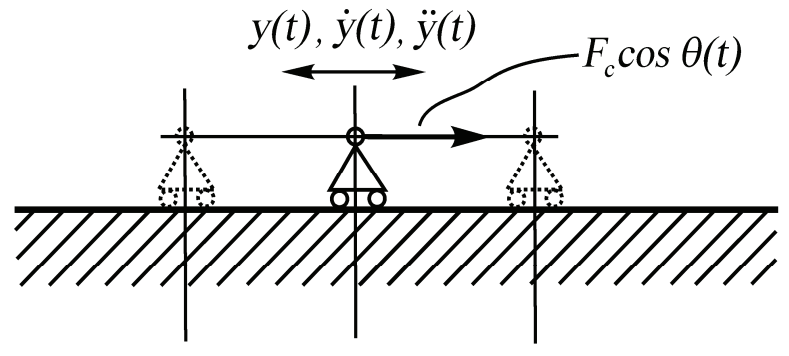

(b)

Fig. 1 (a) A rotating mass $m$ assembled on a slide; (b) maximum oscillation of the slide during the rotation of $m$.

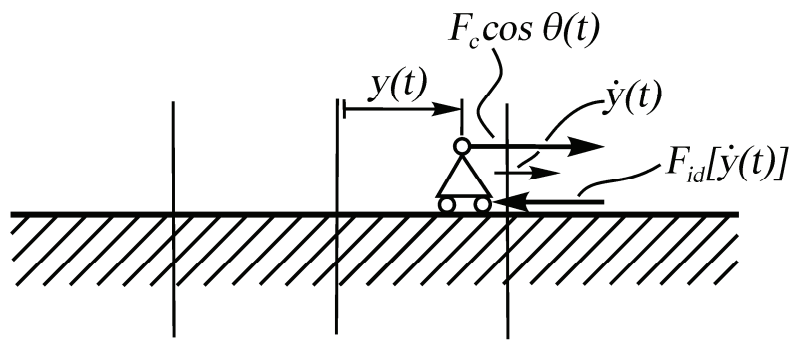

(a)

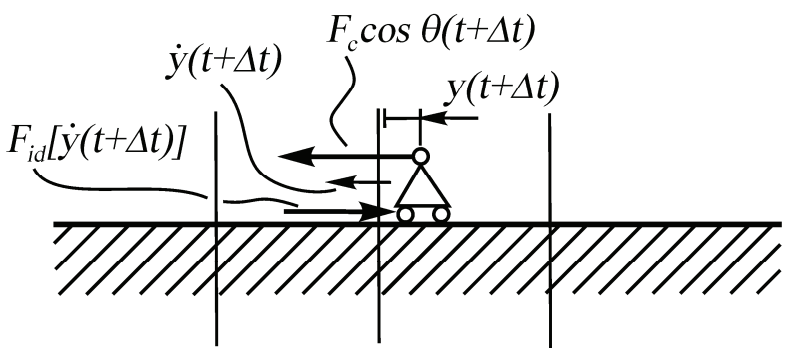

(b)

Fig. 2 (a) Force $F_{i d}[\dot{y}(t)]$ in opposition to $y(t)$; (b) force $F_{i d}[\dot{y}(t+\Delta t)]$ concordant with $y(t+\Delta t)$.

while, at time $t+\Delta t$ this resultant is given by [see Fig. 2(b)]

$$
R(t+\Delta t)=F_{c} \cos \theta(t+\Delta t)-F_{i d}[\dot{y}(t+\Delta t)]
$$

If we assume

$\left|F_{i d}[\dot{y}(t)]\right|<\left|F_{i d}[\dot{y}(t+\Delta t)]\right|$

in relation to the resultant forces defined by Eqs. (1) and (2), one can wonder if it could be

$\mid R(t)]|>| R(t+\Delta t)] \mid$

with $R(t)$ and $R(t+\Delta t)$ oriented to the right and the left, respectively. If condition (4) is true, from $t$ to $t+\Delta t$ the system should move itself to the right. In order to verify if this consideration is effectively correct, we have to proceed by the following quantitative steps: i) to fix the functions $\theta(t)$ and $F_{i d}(\dot{y})$, ii) write the motion equation of the system illustrated in Fig. 1, iii) solve this motion equation, iv) obtain the solution $y(t)$, and v) check that the device moves to the right when $t$ increases.

We observe that $F_{i d}(\dot{y})$ can be considered as a particular constraint reaction that tends to hamper the motion of the device. This impediment depends on the direction of the motion. The impediment is lower and higher when the device is going to the right or to the left, respectively. From a practical point of view and relating to a floating body, we can obtain the function $F_{i d}(\dot{y})$ previously described by constructing correctly the stern and the stem of the hull of the same body. In the next paragraph we illustrate how the system shown in Fig. 1 can be modified and assembled in a hull to produce its translation along a certain direction.

\section{SYSTEM CONFIGURATION AND FUNCTION}

The propulsion mechanism is assembled in the hull of the ship. The mechanism has a pair of counter rotating masses which are subjected to centrifugal forces. By definition, the centrifugal force is an apparent force that presents only when a constraint is defined. The constraint applies the centripetal force to the rotating mass, curving its path, and the corresponding reaction is termed centrifugal force. The centrifugal force is applied to the constraint and stresses it. Hence, this force tends to move the constraint. If the constraint is not able to resist, a displacement of the same constraint occurs. As an example, a body that is totally or partially submerged in water is subject to a reaction of a "partial constraint" defined by the force of hydrodynamic drag. The effect of the centrifugal force on the water is a reaction force. Irrespective of the direction along which the body moves, this force is opposed to the motion of the same body. Consequently, when considering the nature of the body-water relationship, it is reasonable to assume that the "liquid" constraint generates a force opposed to the centrifugal force at any time.

Nevertheless, in opposition to the fixed constraint with a reaction always equal to the centrifugal force (we assume a constant angular velocity), a hull presents a noticeably 
different situation. In hull, the constraint "follows" the motion of the hull. Furthermore, the force of the drag is dependant on the shape and velocity direction of the hull. Hence, by selecting a suitable shape for the front part of the hull, it is possible to minimize the force of hydrodynamic drag when moving forward. Similarly, the rear part of the hull can be shaped in order to obtain the maximum drag force when the hull moves backward. Therefore, on the same module of the relative velocity of the hull (relating to the water), the hydrodynamic drag force will be greater as long as the hull tends to move backward. Then, for example, we can consider two counter rotating equal masses. These masses have the same crank radius and are assembled into the hull. The rotation centre of each crank, and the corresponding rotation, are the same for the both the two masses, at any one time. Consequently, the perpendicular component to the longitudinal axis of the hull relative to the resultant of the two centrifugal forces applied to the corresponding masses is always equal to zero. In contrast, the components of the centrifugal forces, along the longitudinal axis, generate a resultant that reverses its direction every time the cranks are not perpendicular to the longitudinal axis of the hull. When the cranks are horizontally positioned, the resultant of the centrifugal forces is equal to zero. In the other cases, the resultant is different from zero and it is oriented along the direction of the hull system or sterns versus the rotation angle of the cranks. A schematic model of the described system is illustrated in Fig. 3. Whereby $m$ is the counter rotating masses, $r$ the radius of the cranks, $\theta(t)$ the relative angle rotation, $F_{c}$ the centrifugal forces, $\dot{\theta}$ the angular velocity of $m$, and $F_{i d}$ the hydrodynamic drag force that contrasts with the motion of the hull along the $Y$ axis. The reported direction of $F_{i d}$ in Fig. 3 has been fixed based on the assumption of a system motion of the system with velocity $\dot{y}>0$ (where the hull is moving along the positive direction of the $Y$ axis). $F_{i d}$ depends on $\dot{y}$.

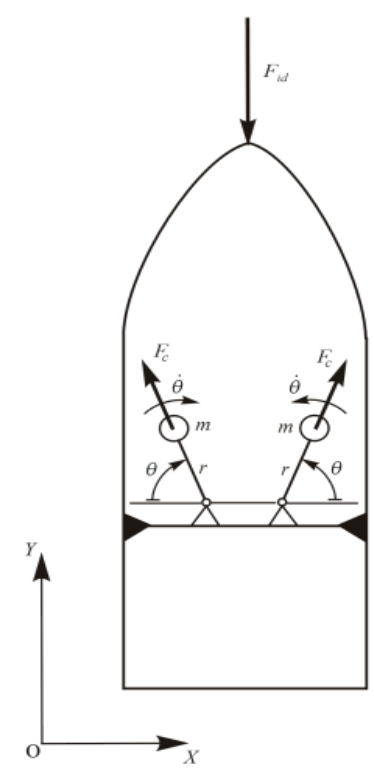

Fig. 3. A hull with a pair of masses that rotate against and hydrodynamic drag force $F_{i d}$.
If $\dot{y}$ is lower than zero, then $F_{i d}$ changes direction and is opposed to the translation of the system along the positive direction of the $Y$ axis. In Fig. 4, a further simplification of Fig. 3 is presented to provide a basic explanation of the system's motion equations that will be completed in the subsequent section. In Fig. 4, $m_{A}$ and $m_{B}$ represent the mass of the hull and the mass that rotates with the angular velocity $\dot{\theta}$.

Since $m_{A}$ is assembled using a slide guide, it can only translate in a vertical direction ( $Y$ axis). The position of $m_{A}$ is identified by the degrees of freedom (DOF) of $y$ that defines the displacement of the hull.

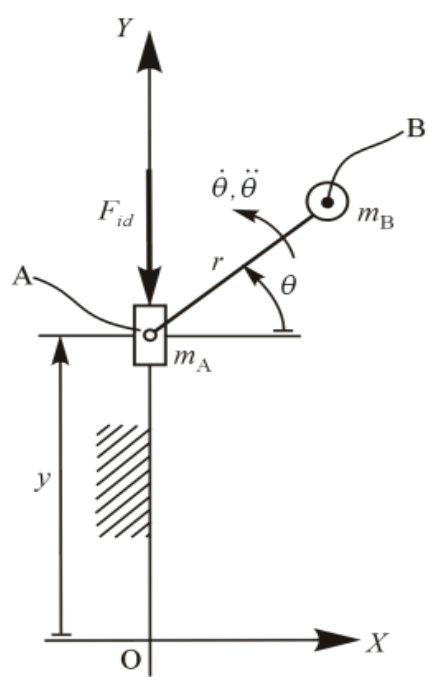

Fig. 4. A system with two degrees of freedom and mass $m_{\mathrm{A}}$ sliding along the $Y$ axis.

The rotating mass $m_{B}$ has a crank radius $r$, the position of which is defined by the angle $\theta$. While this model presents a dynamical equivalent system to that illustrated in Fig. 3, it is simpler because it considers only two masses $m_{A}$ and $m_{B}$. The dynamic equivalence between the two systems can be calculated based on the assumption that $m_{B}=2 \mathrm{~m}$. In the simplified system the external force $F_{i d}(\dot{y})$ is applied to $m_{A}$. This system has two DOF, $y$ and $\theta$.

Based on these systems, in the following sections we will (i) define the motion equations of the system shown in Fig. 4, (ii) formalize the function $F_{i d}$ versus $\dot{y}$, (iii) integrate the previous motion equations, and (iv) evaluate the results that are obtained.

\section{EQUATIONS OF MOTION}

In this section we assess the motion equations of the system illustrated in Fig. 4. Here, $y$ and $\theta$ represent the option of two independent coordinates, which define the two degrees of freedom of the device. In particular, $y$ and $\theta$ can be assumed as the Lagrangian coordinates of the system. Hence, we obtain the above mentioned equations using 
Lagrange's equations. In the case study the two equations are:

$\frac{d}{d t}\left(\frac{\partial E_{c}}{\partial \dot{y}}\right)-\frac{\partial E_{c}}{\partial y}=-F_{i d}$

$\frac{d}{d t}\left(\frac{\partial E_{c}}{\partial \dot{\theta}}\right)-\frac{\partial E_{c}}{\partial \theta}=0$

where $\dot{y}, \dot{\theta}$, and $E_{c}$ represent the velocity of translation of the slide (i.e. of $m_{A}$ ), the angular velocity of the crank, and the kinetic energy, respectively. The only external force $\left(F_{i d}\right)$ of the model is applied towards the $y$ DOF. This is the generalized force that is reported in the second member of equation (5). In this equation, $F_{i d}$ has the minus sign because the generalized forces must fit in Lagrange's equations with plus or minus signs depending on their direction. In other words, if the direction of the force is concordant to that which has been assumed as positive for the relative generalized coordinate, the same force must have a plus sign. If it is the opposite, the force must have a minus sign. Fig. 3 and 4 show that the direction of $F_{i d}$ is opposite to $y$. Therefore the minus sign of $F_{i d}$ in equation (5) is correct. Since the coordinates $x_{B}$ and $y_{B}$ of point $B$, with respect to the reference system $O(X$, $Y)$, are:

$x_{\mathrm{B}}=r \cos \theta$

$y_{\mathrm{B}}=y+r \sin \theta$

and $r$ is a constant that does not change with respect to time, we obtain:

$\dot{x}_{\mathrm{B}}=-r \dot{\theta} \sin \theta$

$\dot{y}_{\mathrm{B}}=\dot{y}+r \dot{\theta} \cos \theta$

Therefore, the kinetic energy of the system is

$$
E_{c}=\frac{1}{2} m_{\mathrm{A}} \dot{y}^{2}+\frac{1}{2} m_{\mathrm{B}}\left(\dot{x}_{\mathrm{B}}^{2}+\dot{y}_{\mathrm{B}}^{2}\right)
$$

which, by Eqs. (9) and (10), becomes

$$
E_{c}=\frac{1}{2}\left(m_{\mathrm{A}}+m_{\mathrm{B}}\right) \dot{y}^{2}+m_{\mathrm{B}} r \dot{y} \dot{\theta} \cos \theta+\frac{1}{2} m_{\mathrm{B}} r^{2} \dot{\theta}^{2}
$$

By computing the partial derivatives the following equations are obtained:

$$
\frac{\partial E_{c}}{\partial \dot{y}}=\left(m_{\mathrm{A}}+m_{\mathrm{B}}\right) \dot{y}+m_{\mathrm{B}} r \dot{\theta} \cos \theta
$$

$\frac{\partial E_{c}}{\partial y}=0$

$\frac{\partial E_{c}}{\partial \dot{\theta}}=m_{\mathrm{B}} r \dot{\theta} \cos \theta+m_{\mathrm{B}} r^{2} \dot{\theta}$

$\frac{\partial E_{c}}{\partial \theta}=-m_{\mathrm{B}} r \dot{y} \dot{\theta} \sin \theta$

This results in the total derivatives:

$\frac{d}{d t}\left(\frac{\partial E_{c}}{\partial \dot{y}}\right)=\left(m_{\mathrm{A}}+m_{\mathrm{B}}\right) \ddot{y}-m_{\mathrm{B}} r \dot{\theta}^{2} \sin \theta+m_{\mathrm{B}} r \ddot{\theta} \cos \theta$

$\frac{d}{d t}\left(\frac{\partial E_{c}}{\partial \dot{\theta}}\right)=m_{\mathrm{B}} r(-\dot{y} \dot{\theta} \sin \theta+\ddot{y} \cos \theta)+m_{\mathrm{B}} r^{2} \ddot{\theta}$

The substitution of equations (17) and (18) in equations (5) and (6) gives the following motion equations of the system illustrated in Fig. 4:

$$
\begin{aligned}
& \ddot{y}+\frac{m_{\mathrm{B}} r}{m_{\mathrm{A}}+m_{\mathrm{B}}}\left(\ddot{\theta} \cos \theta-\dot{\theta}^{2} \sin \theta\right)=-\frac{F_{i d}}{m_{\mathrm{A}}+m_{\mathrm{B}}} \\
& \ddot{y} \cos \theta+r \ddot{\theta}=0
\end{aligned}
$$

\section{Elimination of a generalized coordinate}

The initial status of the system shown in Fig. 3 comprises the following: at time $t=0$ the two cranks are horizontal and do not rotate $[\theta(0)=0, \dot{\theta}(0)=0]$. Subsequently, at $t>0$ the cranks begin to rotate and their angular velocity $\dot{\theta}(\mathrm{t})$ changes versus time in accordance with a specific law. This law can be defined by the first phase of acceleration, with a generally constant working speed, and a final step where the velocity $\dot{\theta}(t)$ decreases to zero. However, in relation to this hypothetical working cycle, the function $\theta(t)$ is fixed a priori. From an experimental point of view, we can establish that $\theta(t)$ changes by governing the engine assembled in the hull. This engine produces the rotation of the masses $m$ illustrated in Fig. 3. In relation to these observations, in Eqs. (19) and (20) $\theta(t)$ is no longer an independent coordinate. Therefore, the motion of the system illustrated in Fig. 4 is dependent on the laws $\theta(t), \dot{\theta}(t)$, and $\ddot{\theta}(t)$ that have been fixed a priori. Consequently, regarding the motion of the masses $m_{\mathrm{A}}$ and $m_{\mathrm{B}}$, equation (20) relative to the DOF $\theta$ must not be considered. Hence, the only equation that draws the motion of the system showed in Fig. 4 is represented by Eq. (19). We can rewrite this ordinary differential non-homogeneous equation with a variable coefficient by pointing out the dependence on time of the same coefficient $\ddot{\theta} \cos \theta-$ $\dot{\theta}^{2} \sin \theta$ : 


$$
\ddot{y}+\frac{m_{\mathrm{B}} r}{m_{\mathrm{A}}+m_{\mathrm{B}}}\left[\ddot{\theta}(t) \cos \theta(t)-\dot{\theta}(t)^{2} \sin \theta(t)\right]=-\frac{F_{i d}(\dot{y})}{m_{\mathrm{A}}+m_{\mathrm{B}}}
$$

\section{Function $\theta(t), \dot{\theta}(t)$, and $\ddot{\theta}(t)$ fixed}

In this study, to establish $\theta(t)$ it can be convenient to fix first the function $\dot{\theta}(t)$. As a result of the integration and derivation of $\dot{\theta}(t)$ with respect time $t$, the corresponding functions $\theta(t)$ and $\dot{\theta}(t)$ are obtained. Regulation of the angular velocity of the engine with time, produces the rotation of $m$, as would occur in a real test. Furthermore, for the effective management of the numerical integration of the motion equation (21), the function $\dot{\theta}(t)$ was selected to be continuous. To improve simplicity and reliability the entire analytical formulation, $\dot{\theta}(t)$ was obtained starting from harmonic functions. Furthermore, these functions have been managed and modified by methods similar to those utilized for studying the motion laws of the cams. In this way, simple analytical functions with respect to adjacent domains have been obtained. These functions were effectively managed and utilized by algebraic integration software (Mathematica), through which any tests were performed. In Figs. 5 and 6 two examples of functions $\theta(t), \dot{\theta}(t)$, and $\ddot{\theta}(t)$ utilized in equation (21) are presented, in addition to the adjacent domains. In Appendix A the general expressions of these functions are reported.

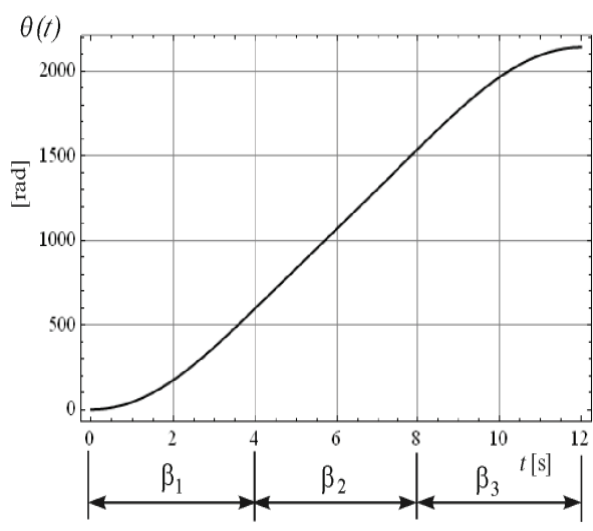

(a) Rotation angle $\theta$

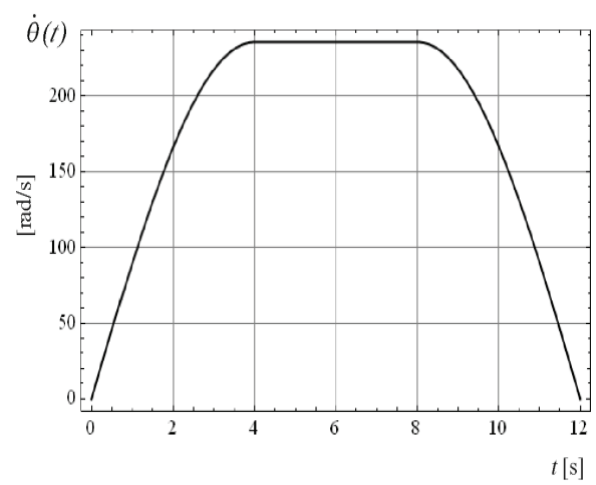

(b) Angular velocity $\dot{\theta}$

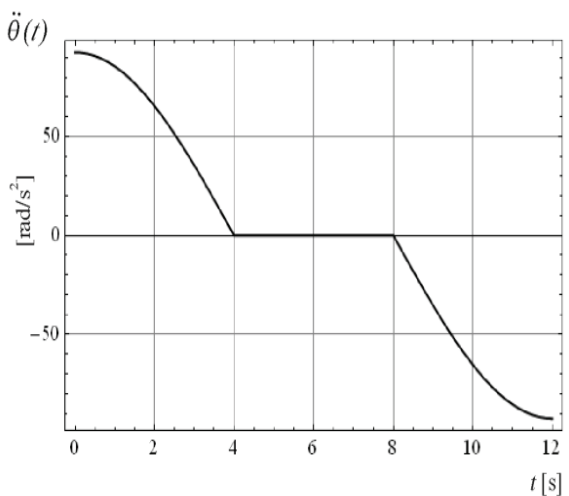

(c) Angular acceleration $\ddot{\theta}(t)$

Fig. 5. (a) Rotation angle $\theta$, (b) angular velocity $\dot{\theta}$, and (c) angular acceleration $\ddot{\theta}(\mathrm{t})$ of the mass $m_{B}$ with increasing, stationary, and decreasing work cycle versus time.

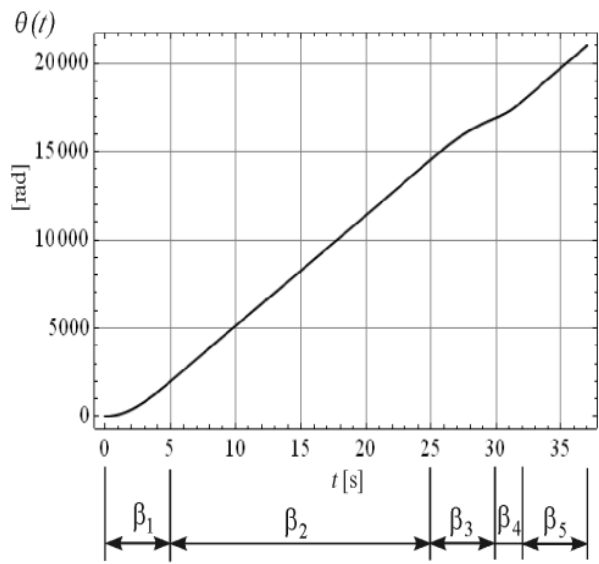

(a) Rotation angle $\theta$

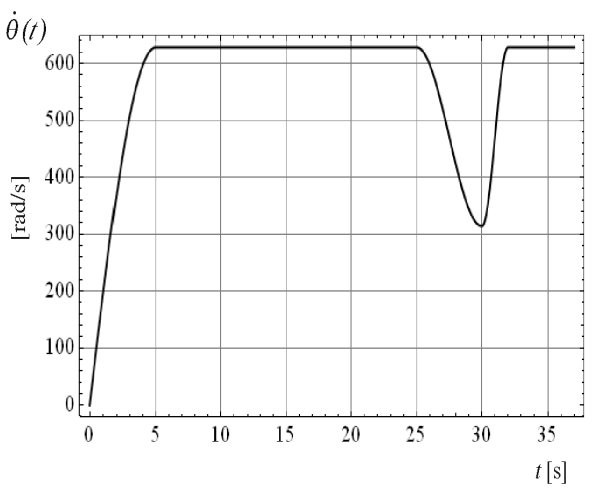

(b) Angular velocity $\dot{\theta}$

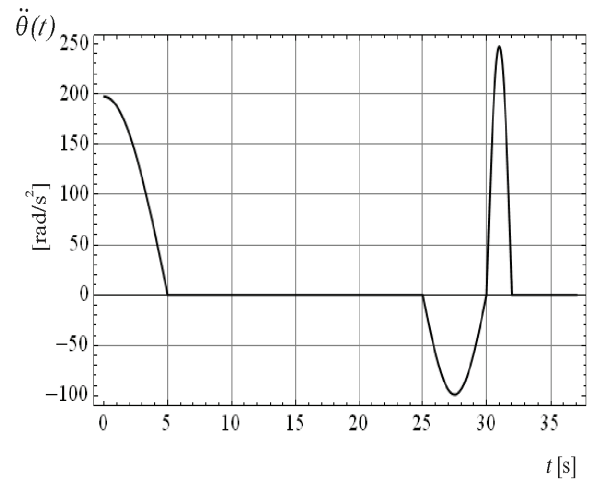

(c) Angular acceleration $\ddot{\theta}(t)$

Fig. 6. (a) Rotation angle $\theta$, (b) angular velocity $\dot{\theta}$, and (c) angular acceleration $\ddot{\theta}(\mathrm{t})$ of the mass $m_{B}$ with increasing, stationary, decreasing, new increasing, and new decreasing work cycle versus time. 
The domains are denoted by $\beta_{i}(i=1,2, \ldots, 5)$. The time $t_{i}(i=1,2, \ldots, 5)$ which defines the range $\beta_{i}$, is given by the following equations:

$$
\begin{aligned}
& t_{1}=\beta_{1} \\
& t_{2}=\beta_{1}+\beta_{2} \\
& t_{3}=\beta_{1}+\beta_{2}+\beta_{3} \\
& t_{4}=\beta_{1}+\beta_{2}+\beta_{3}+\beta_{4} \\
& t_{5}=\beta_{1}+\beta_{2}+\beta_{3}+\beta_{4}+\beta_{5}
\end{aligned}
$$

\section{Generalized force $\boldsymbol{F}_{i d}(\dot{\boldsymbol{y}})$}

The force $F_{i d}(\dot{y})$ is a function of the velocity $\dot{y}$ relative to the mass $m$. In order to define $F_{i d}(\dot{y})$, a range of simplified hydrodynamic simulations have been conducted. Specifically, three bodies were modeled (SolidWorks), including (i) a two dimensional body (i.e. a section of a hull of a hypothetical boat) completely surrounded by water; the sectioning is performed by a plane passing for the line of the buoyancy (Fig. 7), (ii) a three dimensional body partially submerged in water, representing a simplified version of an actual boat (Fig. 8 ), and (iii) a three dimensional body totally submerged in water, similar to that of a bullet (Fig. 9).

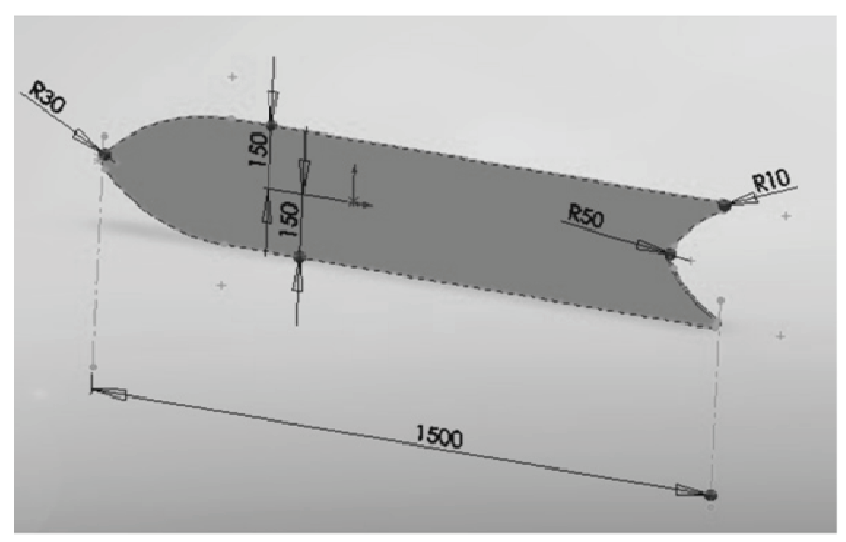

Fig. 7. CAD model of a two dimensional body completely surrounded by water.

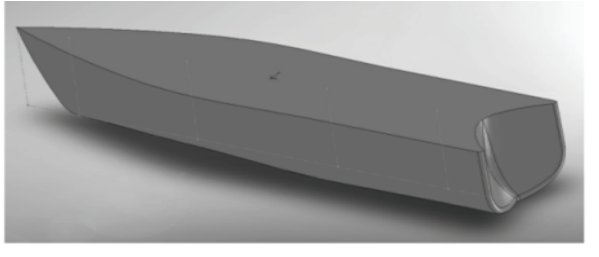

(a) CAD model of a three dimensional boat.

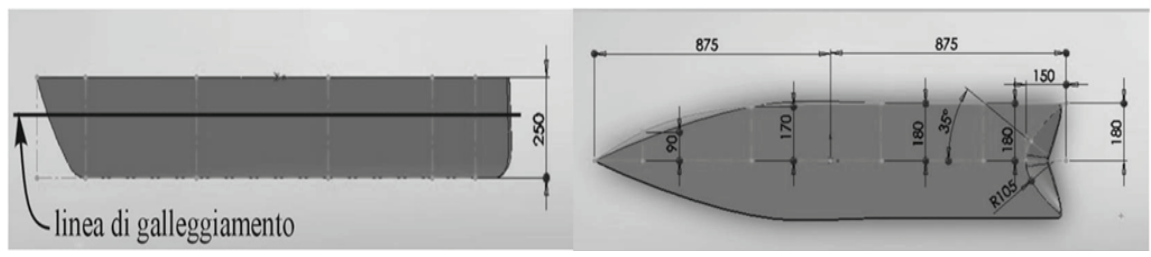

(b) Relative dimensions.

Fig. 8. (a) CAD modelization of a three dimensional model boat-like body partially submerged in water, and (b) the relative dimensions in $\mathrm{mm}$.

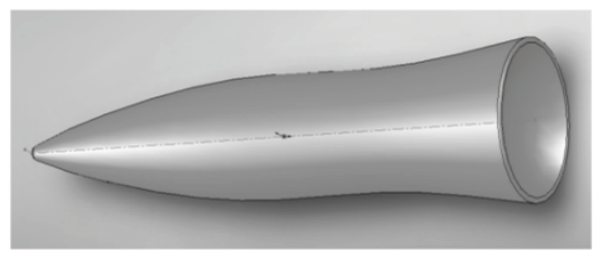

(a) CAD model of a three dimensional boat.

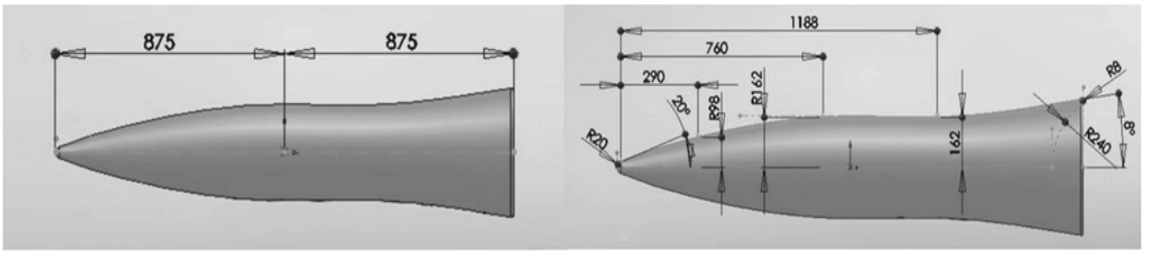

(b) Relative dimensions.

Fig. 9. (a) CAD model of a three dimensional bullet-like body completely submerged in water, and (b) the relative dimensions.

Each of these structures was modelled to have a different hydrodynamic drag force with respect to the direction along which the body translates on the module $|\dot{y}|$ of the velocity $\dot{y}$. By using an available fluid dynamic software simulation (CosmosFloWorks), we only computed the hydrodynamic drag force relative to the viscosity of the water. Fig. 10 shows three examples of flow stream visualizations obtained in relation to the three described body types. The longitudinal dimension of these structures varies from 1.5 to $1.75 \mathrm{~m}$. The more realistic and reliable data of drag force obtained by the previous fluid dynamic simulations are those that corresponded to the completely submerged three dimensional body (Fig. 9 and 10(c)). In fact, the first hull model type (Fig. 7) was primarily developed to verify the computation time and difference in force $F_{i d}(\dot{y})$ versus the velocity $\dot{y}$ direction. The second model type (Fig. 8) was designed to provide an approximation of real fluid dynamic behavior.

To simplify the simulation, it was assumed that the upper side of the fluid domain is defined by the plane passing for the line of the buoyancy (Fig.8(b)). This means that the 
surface wave-motion generated by the hull was not correctly simulated. However, surface waves are small when the velocity is not too high. Despite this, these waves waste energy and increase the drag force that opposes the motion of the body. Therefore this force does not depend only on the viscosity of the water. Consequently, the main limitation of model (ii) is where the drag of the wave force versus $\dot{y}$ was neglected (Couser et al. 1998). From a fluid dynamic simulation perspective, model (iii) was considered to be the optimal hull body type. This is because the body is completely submerged underwater; hence simulation of drag caused by surface waves have not to be performed.

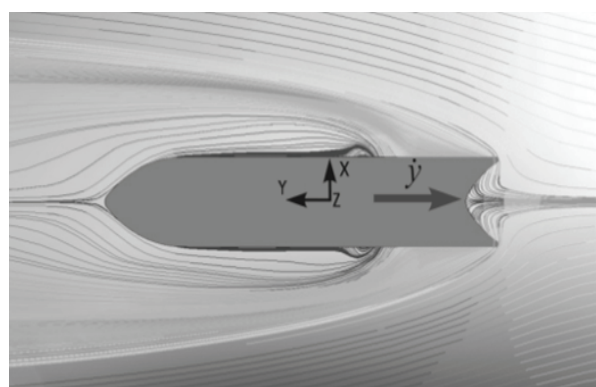

(a)

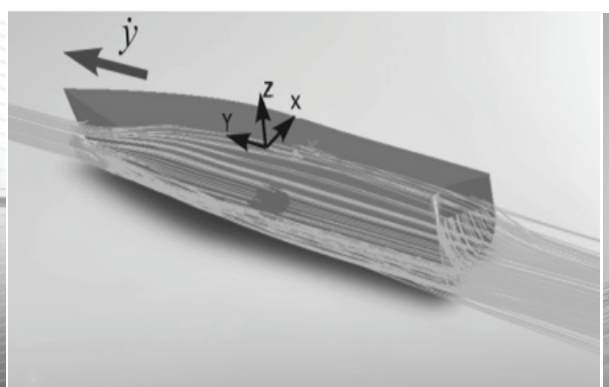

(b)

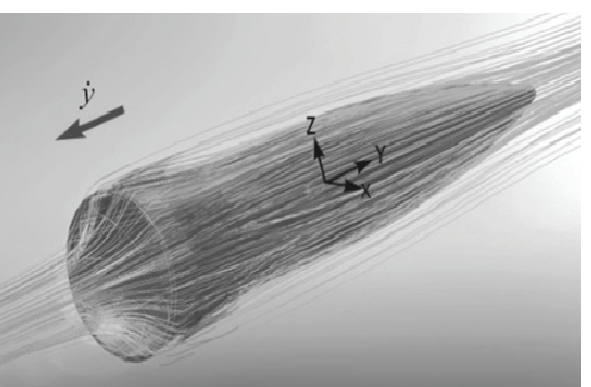

(c)

Fig. 10. Flow streams relative to (a) the two dimensional body completely submerged in water moving with velocity equal to $-8.34 \mathrm{~m} / \mathrm{s}$, (b) the partially submerged three dimensional boat-like body moving with velocity equal to $5.56 \mathrm{~m} / \mathrm{s}$, and (c) the completely submerged three dimensional bullet-like body moving with velocity equal to $-1.39 \mathrm{~m} / \mathrm{s}$.

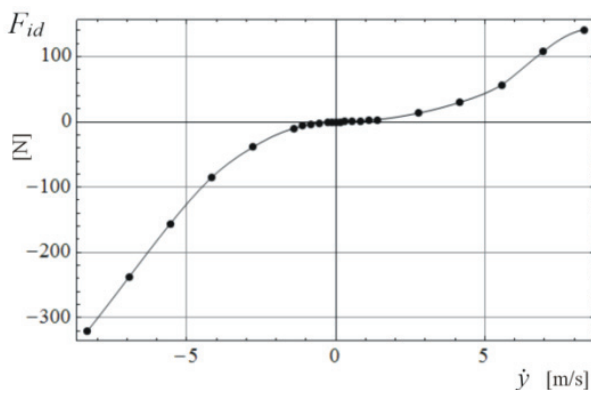

(a)

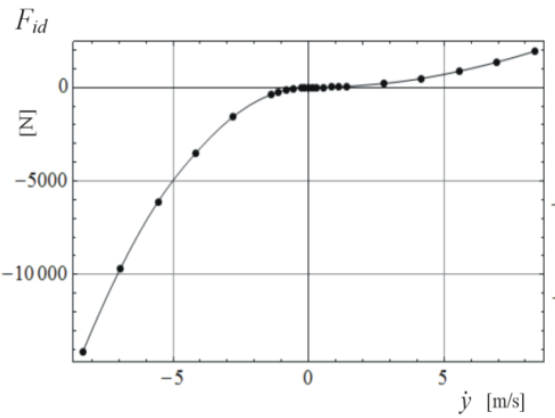

(b)

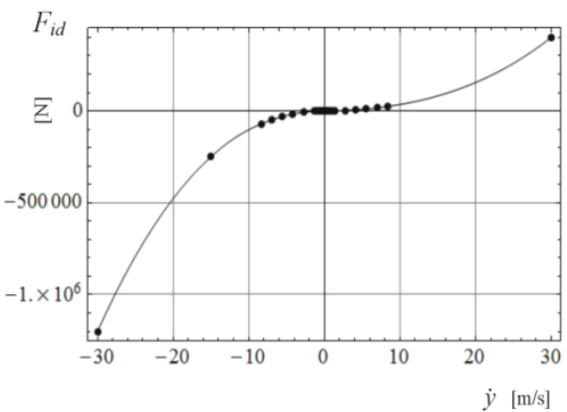

(c)

Fig. 11. Hydrodynamic drag force $F_{i d}$ versus the velocity $\dot{y}(t)$ (a) for the two dimensional body completely submerged, (b) the partially submerged three dimensional boat-like body, and (c) the completely submerged three dimensional bullet-like body.

Fig. 11 shows the three functions of $F_{i d}(\dot{y})$ obtained in relation to the three body types illustrated in Fig. 7-8. Each point on the graphs defines the results obtained by the corresponding simulation, performed with defined constant velocities $\dot{y}$. The continuous curve of best fit that interpolates the points is an interpolating polynomial of third degree (spline). Hence, whatever the value of $\dot{y}$, the corresponding value of $F_{i d}$ in the interpolation domain can be determined. For model (iii), the points reported in Fig. 10(c) correspond to the results obtained by the fluid dynamic simulations. In this graph, the first two points and the last point have been added on the basis of a simple estimated extrapolation of $F_{i d}(\dot{y})$.

The purpose of extrapolating model (iii) was to extend the definition domain relative to the interpolation of $F_{i d}$, without too large increase in the number of the fluid dynamic simulations. As a result, the value of $F_{i d}$ can be assed from -30 to $+30 \mathrm{~m} / \mathrm{s}$, which is a very high velocity for a motion body in water. While it may be possible to assume that these values of velocity may be achieved during experimental tests, phenomena that are not correctly foreseen by the fluid dynamic software may arise. For this reason the addition of the three extrapolate points in Fig. 11c help improve the ease of computing high enough values of $F_{i d}$ in this study, that may not necessarily be close or equal to -30 or $+30 \mathrm{~m} / \mathrm{s}$. The necessity to consider such a wide domain exists due to the numerical integration of the motion equation of the body submerged in water.

In fact, the values computed of $\dot{y}$ were not always included in the domain defined by the only points relative to the fluid dynamic simulations previously performed. Since many formulations of the motion equations have been 
performed by varying the parameters of the system, the maximum (or the minimum) value that $\dot{y}$ has in each integration is unknown a priori. Therefore, in order to avoid executing numerous new fluid dynamic simulations to continuously update the domain of $\dot{y}$, we selected to proceed as previously described. However, the unusually high values of $\dot{y}$, computed by the utilized software (Mathematica), were always isolated. It is highly probable that these high values may depend on the method that was implemented by the software control functions in the algorithm of integration. In fact, with the exception of the high peaks associated to the previously mentioned values, the results of the integrations always showed regular trends and reasonable amplitudes.

\section{RESULTS}

Equation (21) was numerically integrated by fixing the initial conditions $y(0)$ and $\dot{y}(0)$ at time $t=0$, with the laws $\theta(t), \dot{\theta}(t)$, and $\ddot{\theta}(t)$ reported in Appendix A. In particular, in order to summarize at least a part of the results obtained, equation (21) can be written as:

$$
\ddot{y}+\frac{r}{k+1}\left[\ddot{\theta}(t) \cos \theta(t)-\dot{\theta}(t)^{2} \sin \theta(t)\right]=-\frac{F_{i d}(\dot{y})}{m_{\mathrm{B}}(k+1)}(27)
$$

where $\quad k=\frac{m_{\mathrm{A}}}{m_{\mathrm{B}}}$

varies at certain ranges. Since the performed numerical integrations indicate that the $y(t)$ displacement is highly dependents on the ratio $k$ under $r$, the formulation of the motion equation defined by equation (27) was adopted. Fig. 12 shows a graph of maximum displacement $y_{\mathrm{MAX}}$ after $40 \mathrm{~s}$ of operation at $0.1 \leq k \leq 10$ and $m_{B}=2 \mathrm{Kg}$, for the system illustrated in Fig. 4. This spline polynomial curve was interpolated by performing the integration for the values of $k$ represented by the points in Fig. 12 only. The results synthesized by the spline curve were acquired by fixing accelerating, stationary, and decelerating $\dot{\theta}(t)$ cycles analogous to those reported in Fig. 5. In this instance, the body type used is model (iii), the completely submerged three dimensional bullet-like body (Fig. 9). With respect to $F_{i d}(\dot{y})$, the function shown in Fig. 11(c) has been utilized. The numerical values of parameters $\beta_{i}(i=1,2,3), r$, and $h$ used in this simulation are indicated in Table 1 (and Appendix equations A1-A4). This table shows that the selected value of $h$ corresponds with an angular stationary velocity $\dot{\theta}_{R}(t)$ equal to $6000 \mathrm{rpm}$.

Table 1. Parameters of the laws $\theta(\mathrm{t}), \dot{\theta}(\mathrm{t})$, and $\ddot{\theta}(\mathrm{t})$ to obtain the data shown in Fig. 12.

\begin{tabular}{|l|c|c|c|c|c|c|}
\hline$\beta_{1}(\mathrm{~s})$ & $\beta_{2}(\mathrm{~s})$ & $\beta_{3}(\mathrm{~s})$ & $r(\mathrm{~m})$ & $h$ & \multicolumn{2}{|c|}{$\dot{\theta}_{R}$} \\
\hline 10.0 & 20.0 & 10.0 & 0.09 & 4000.0 & 628.319 & 6000.0 \\
\hline
\end{tabular}

Table 2. Parameters of the laws $\theta(t), \dot{\theta}(t)$, and $\ddot{\theta}(t)$ shown in Fig. 6.

\begin{tabular}{|l|c|c|c|c|c|c|c|c|}
\hline$\beta_{1}$ & $\beta_{2}$ & $\beta_{3}$ & $\beta_{4}$ & $\beta_{5}$ & $r$ & & \multicolumn{2}{|c|}{$\dot{\theta}_{R}$} \\
$(\mathrm{~s})$ & $(\mathrm{s})$ & $(\mathrm{s})$ & $(\mathrm{s})$ & $(\mathrm{s})$ & $(\mathrm{m})$ & $h$ & $(\mathrm{rad} / \mathrm{s})$ & $(\mathrm{rpm})$ \\
\hline 5.0 & 20.0 & 5.0 & 2.0 & 5.0 & 0.09 & 2000 & 628.319 & 6000 \\
\hline
\end{tabular}

The curve shown in Fig. 12 indicates that the lower the value of $m_{A}$ with respect $m_{B}$, the higher the corresponding displacement $y_{M A X}$. This trend was confirmed for all the other simulations performed in this study. Moreover, all obtained $y(t)$ functions were always modulated by a frequency dependant on angular velocity $\dot{\theta}(t)$. Nevertheless, the amplitude of this modulation may also be very small, and the function $y(t)$ results almost always increased. The tests indicated that oscillating trends were observed in relation to $\dot{y}(t)$. The values of these oscillations were both positive and negative; however the mean value of $\dot{y}(t)$ was always positive. Therefore, the simulations showed that the body moves in a positive direction along the $Y$ axis, even when vibrating. The acceleration $\ddot{y}(t)$ is also characterized by an oscillating trend, but the mean value is almost equal to zero.

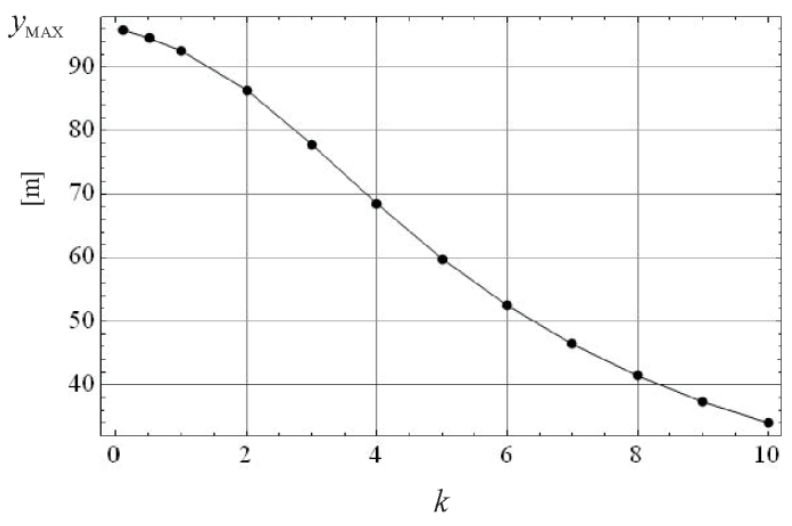

Fig. 12. Maximum displacement $y_{\mathrm{MAX}}$ versus the ratio $k=m_{\mathrm{A}} / m_{\mathrm{B}}$ with $m_{\mathrm{B}}=2 \mathrm{Kg}$.

These results were also obtained by fixing different $\theta(t)$, $\dot{\theta}(t)$, and $\ddot{\theta}(\mathrm{t})$ functions, provided these functions had trends similar to those illustrated in Figs. 5 and 6. An example of $y(t), \dot{y}(t)$, and $\ddot{y}(t)$ functions from performing the numerical integration of equation (27) for model (iii) is reported in Fig. 13. In this simulation the parameter reported in Table 1 was adopted with the values of $m_{A}$ and $m_{B}$ being equal to 20 and 4 $\mathrm{Kg}$, respectively. From Fig. 13(b) graph of $\dot{y}(t)$ it was found the average velocity is always positive and, in the range where $\dot{\theta}(t)$ is constant, this velocity is approximately equal to $0.6 \mathrm{~m} / \mathrm{s}$. In reference to the curves of $\dot{y}(t)$ and $\ddot{y}(t)$, the oscillations frequency is shown to be directly correlated to the value of the angular velocity $\dot{\theta}(t)$, with which $m_{B}$ rotates. In particular, in the region where $\dot{\theta}(t)$ is constant, $\dot{y}(t)$ and $\ddot{y}(t)$ are periodic with a frequency equal to $100 \mathrm{~Hz}$. This 
frequency only corresponds with the stationary angular velocity of $6000 \mathrm{rpm}$ of $m_{\mathrm{B}}$ fixed for performing the simulation. Fig. 14 shows the functions $y(t), \dot{y}(t)$, and $\ddot{y}(t)$ for $15.0 \leq t \leq 15.0$ seconds. Here, the periodicity of the functions is clearly indicated. This periodicity is also noticeable for the curve $y(t)$.

This particular trend of $y(t)$ is not clear in Fig. 13(a) (showing a straight line) because the amplitude of the oscillation was too small. Analogous results were obtained when considering (i) different $\theta(t)$ functions with respect to those shown in Fig. 5(b), and (ii) the first two models for which the curves $F_{i d}(\dot{y})$ were assessed (Figs. 7, 8, 10(a, b) and $11(\mathrm{a}, \mathrm{b})$. In the example of model (iii), the use of the functions $\theta(t), \dot{\theta}(t)$, and $\ddot{\theta}(t)$ reported in Fig. 6 (and equations A5-A8), $y(t), \dot{y}(t)$, and $\ddot{y}(t)$ alter as shown in Fig. 15. As in Table 1, Table 2 shows the corresponding simulation parameters utilized for defining the laws $\theta(t)$, $\dot{\theta}(t)$, and $\ddot{\theta}(t)$ that establish the rotation of $m_{\mathrm{B}}$. In this case $m_{\mathrm{A}}=20 \mathrm{Kg}$ and $m_{\mathrm{B}}$ have again been fixed to be equal to 2 $K g$.

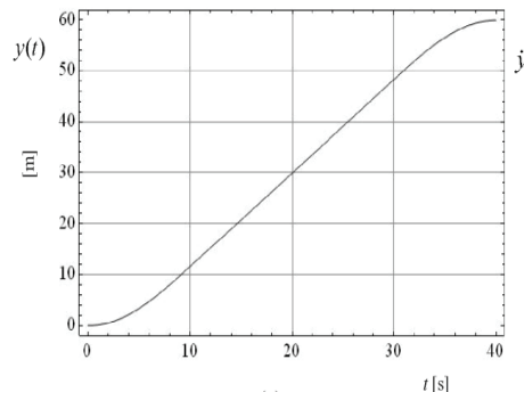

(a) Displacement $y(t)$

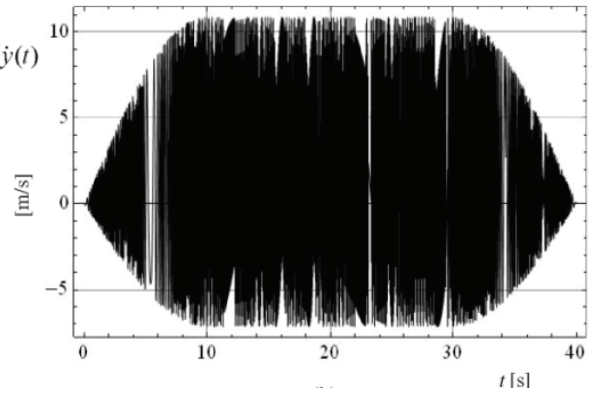

(b) Velocity $\dot{y}(t)$

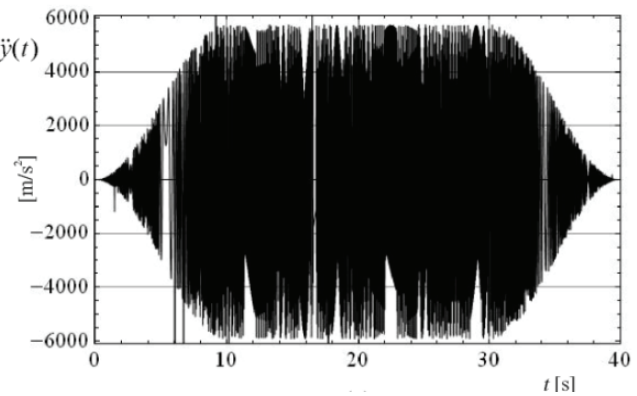

(c) Acceleration $\ddot{y}(t)$

Fig. 13. An example of (a) displacement $y(t)$, (b) velocity $\dot{y}(t)$, and (c) acceleration $\ddot{y}(t)$ for the completely submerged three dimensional bullet-like body with work cycle versus time of the angular velocity of mass $m_{\mathrm{B}}$ when accelerating, stationary, and decelerating.

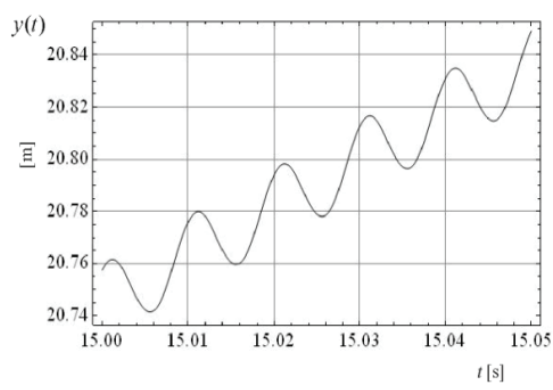

(a) Displacement $y(t)$

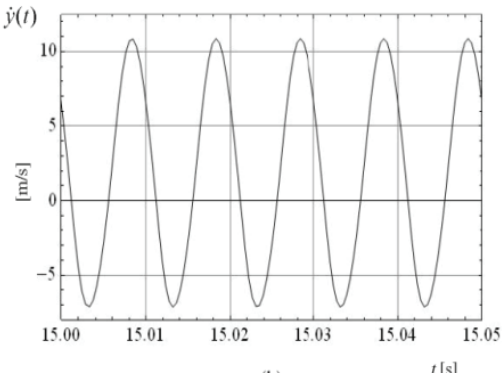

(b) Velocity $\dot{y}(t)$

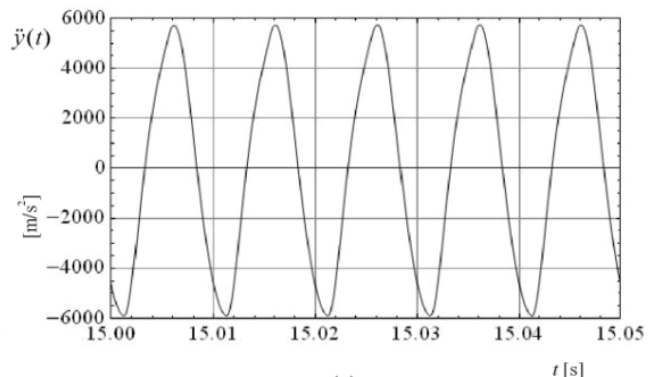

(c) Acceleration $\ddot{y}(t)$

Fig. 14. The periodicity of (a) the displacement $y(t)$, (b) the velocity $\dot{y}(t)$, and (c) the acceleration $\ddot{y}(t)$ for the completely submerged three dimensional bullet-like body, with constant angular velocity of the mass $m_{\mathrm{B}}$.

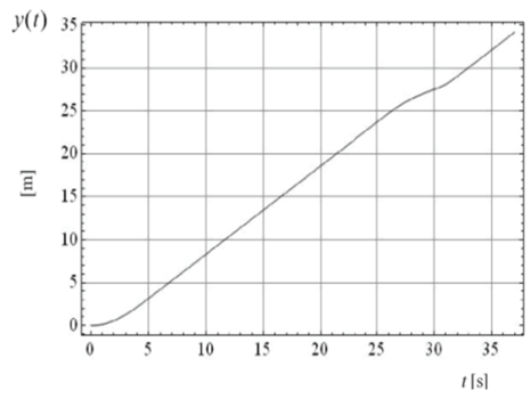

(a) Displacement $y(t)$

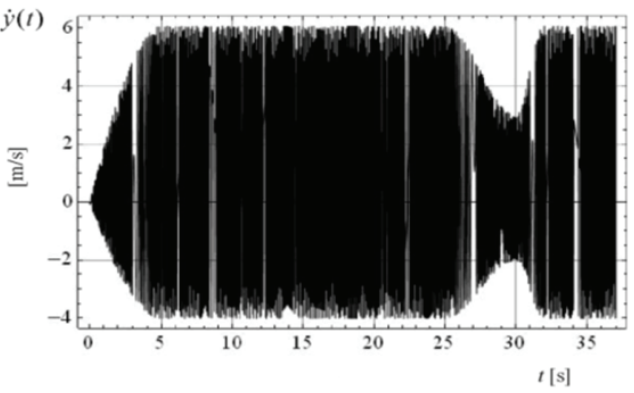

(b) Velocity $\dot{y}(t)$

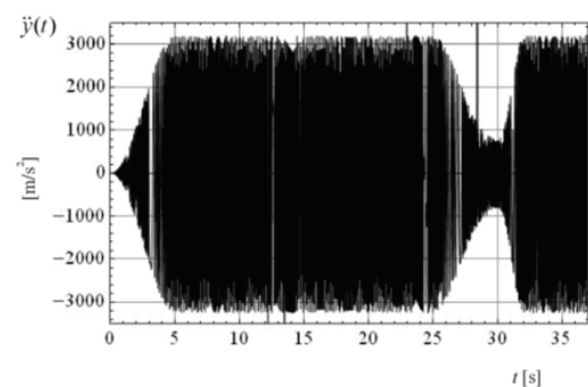

(c) Acceleration $\ddot{y}(t)$

Fig. 15. (a) Displacement $y(t)$, (b) velocity $\dot{y}(t)$, and (c) acceleration $\ddot{y}(t)$ for the completely submerged three dimensional bullet-like body, with a working cycle of mass $m_{\mathrm{B}}$ illustrated in Fig. 6 . 
In order to clearly verify that the motion of the system along the positive direction of the $Y$ axis depends only on the difference of the hydrodynamic drag forces versus hull motion direction, a hypothetical anti-symmetric function $F_{i d}(\dot{y})$ was considered, which is shown in Fig. 16. The function was obtained by using the points that represent $F_{i d}(\dot{y})$ (Fig. 11(c)) where the abscissa vary from 0 to $10.0 \mathrm{~m} / \mathrm{s}$. The anti-symmetric function $F_{i d}(\dot{y})$ was obtained by changing the sign of the abscissas and ordinates relative to these points, for reporting in the Cartesian plane. For example, this new function could characterize a pseudo-cylindrical body, with similarly shaped ends. Fig. 17 shows the integration of the motion equation of the system with the functions functions $\theta(t), \dot{\theta}(t)$ and $\dot{\theta}(t)$ (Fig. 5), $m_{\mathrm{A}}=20 \mathrm{Kg}, m_{\mathrm{B}}=2 \mathrm{Kg}$, and the antisymmetric $F_{i d}(\dot{y})$. The graph of $y(t)$ shows that model (iii) always oscillates around its initial position throughout the working cycle, fixed by the function $\theta(t)$. As a result, the mean velocity is zero. Analogous results were obtained when performing other simulations with an antisymmetric curve $F_{i d}(\dot{y})$ different to that shown in Fig. 16. Hence we can establish that the prevailing motion of the system, in a certain direction, is dependent on the different action of the constraint in one direction with respect to the opposite direction. If this action is equal for both directions, the system only oscillates. In contrast, if the system continues along the direction where the constraint generates a lower reaction, this is the direction where the hydrodynamic drag force is lower.

On the contrary, the system goes on along the direction where the constraint generates a lower reaction, that is along the direction where the hydrodynamic drag force is lower.

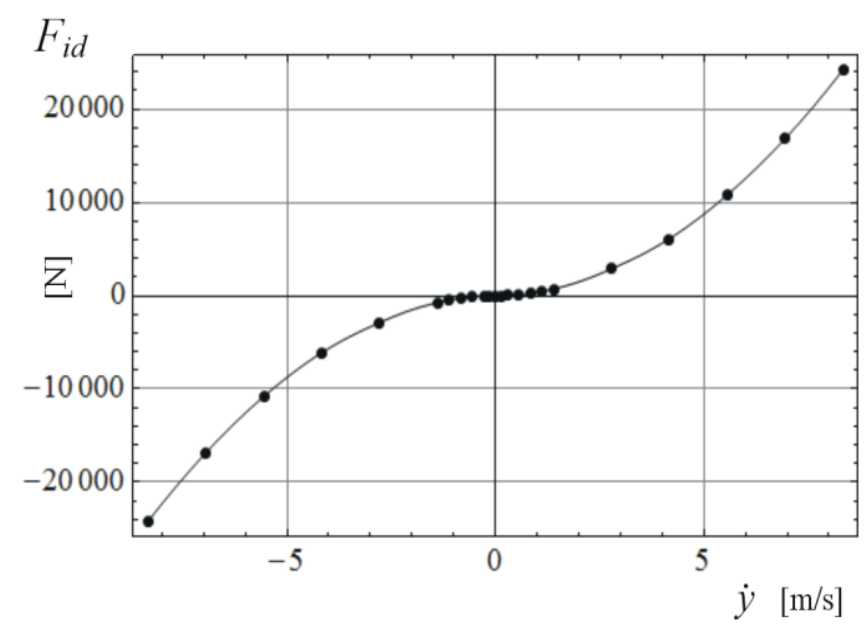

Fig. 16 Hypothetical anti-symmetric hydrodynamic drag force $F_{i d}$ versus velocity $\dot{y}$ for the completely submerged three dimensional bullet-like body

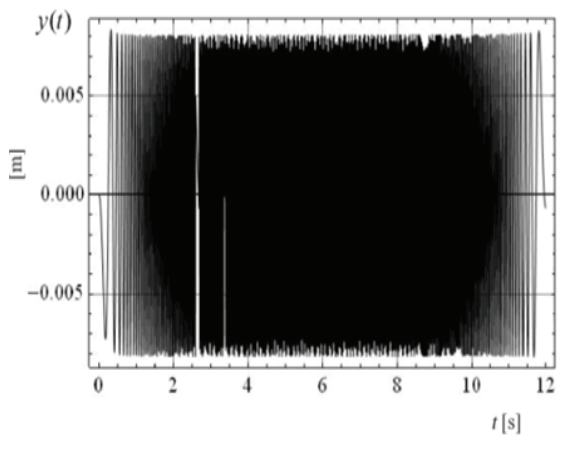

(a) Displacement $y(t)$

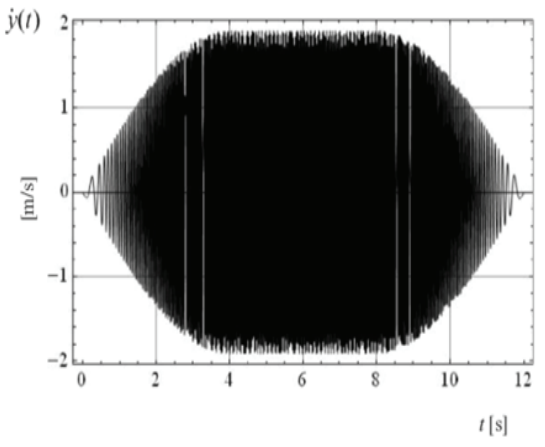

(b) Velocity $\dot{y}(t)$

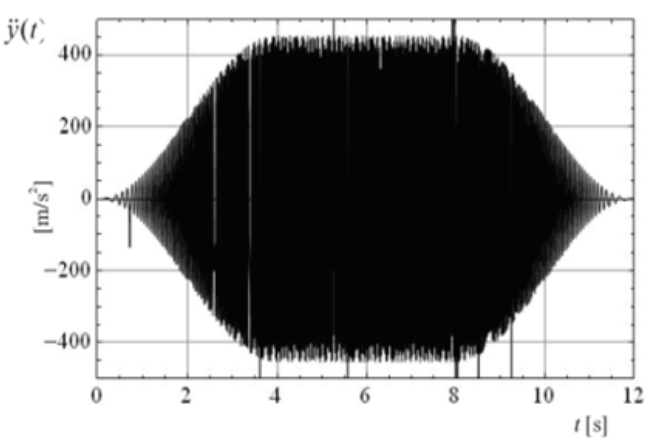

(c) Acceleration $\ddot{y}(t)$

Fig. 17 (a) Displacement $y(t)$, (b) velocity $\dot{y}(t)$, and (c) acceleration $\ddot{y}(t)$ for the completely submerged three dimensional bullet-like body with anti-symmetric hydrodynamic drag force $F_{i d}$.

\section{CONCLUSIONS}

This study confirms through numerical analyses and simulations the potential to obtain rectilinear displacements, controlled along a certain direction, by using pairs of masses that counter rotation. The condition that requires fulfiling, is defined by a particular constraint applied to the system. This constraint must generate different reaction forces with respect to the two rectilinear directions along which the system must translate. The simulations clearly illustrate this behavior. The computations were executed with reference to bodies that were surrounded, partially or completely submerged in water, where we assembled a device similar to that shown in Fig. 1. In this context, the function $F_{i d}(\dot{y})$ represented the reaction of the constraint opposed to the motion of translation. $F_{i d}(\dot{y})$ was obtained through fluid dynamic simulations. These 
simulations were approximations, however were deemed acceptable for the function $F_{i d}(\dot{y})$ from a semi-quantitative perspective, at least to simulate a reaction of the constraint comprising different values with respect to the motion direction of the hull. The values of the masses and the functions of the angular velocity, by which the eccentric mass rotates, were selected to provide a close representation of a possibly real experiment, in order to verify the numerical results obtained. From an engineering perspective, the construction of a floating model, with length equal to $1.75 \mathrm{~m}$, is attainable. The problem of the eccentric masses assembled within the hull, and rotating with an angular velocity equal to several thousands of rpm, can also be easily resolved by using more than one device, each with smaller masses $m_{B}$ and radii $r$. With respect to the displacement maximization, all analyses that were performed could be formalized as an optimization problem, where the optimization variables are the masses, the radii of rotation, and the parameters that define the function $\dot{\theta}(t)$. With respect to the problem of vibration, the simulations clearly indicate that the hull will vibrate primarily along its longitudinal direction. The frequency of these vibrations is directly associated to the angular velocity of the rotating masses. Nevertheless, the simulation models remain too basic to properly evaluate the inconvenience that may arise from the propagation of the vibrations, particularly in reference to a real boat equipped by the suggested propulsion system. Therefore, if the results obtained by the simulations performed in this study are sufficiently confirmed by experimental tests, the potential vibration problem would require consideration.

\section{REFERENCES}

Ressel, J., 1827, Austrain Patent, A never-ending screw which can be used to drive ships both on sea and rivers.

Ericsson, J., 1838, U.S. Patent 588, Propelling Steam Vessels Screw Propeller. United States Patent 588 1.2.1838.

Smith, F.P., 1836, English Patent, Archimedean Screw Propeller.

Colombo, G., Manuale dell'ingegnere, 1985, p. G-152, Ed. Ulrico Hoepli, 81a edizione, Milano, 1985.

Colombo, G., Manuale dell'ingegnere, 1895, p. G-147, Ed. Ulrico Hoepli, 81a edizione, Milano, 1985.

Mathematica, Wolfram Research, Champaign, USA, IL WorldwideHeadquarters,

http://www.wolfram.com/products/mathematica/index.ht $\mathrm{ml}$

SolidWorks, Dassault Systèmes SolidWorks Corporation, http://www.solidworks.com/

CosmosFloWorks, SRAC, SolidWorks Corporation, http://www.cosmosm.com/

Couser, P. Wellicome, J. F. and Molland, A. F., 1998. An Improved Method for the Theoretical Prediction of the Wave Resistance of Transom Stern Hulls using a Slender Body Approach, International Shipbuilding Progress, 45(444), 1998.

\section{APPENDIX}

Functions $\theta(t), \dot{\theta}(t)$, and $\ddot{\theta}(t)$ utilised to obtain the graphics illustrated in Fig. 5:

$$
\theta(t)=\left\{\begin{array}{cc}
2 h \sin \left(\frac{\pi t}{4 \beta_{1}}\right)^{2} & ; 0 \leq t<t_{1} \\
2 h \sin \left(\frac{\pi t_{1}}{4 \beta_{1}}\right)^{2}+\frac{h \pi\left(t-t_{1}\right) \sin \left(\frac{\pi t_{1}}{2 \beta_{1}}\right)}{2 \beta_{1}} & ; t_{1} \leq t<t_{2} \\
2 h \sin \left(\frac{\pi t_{1}}{4 \beta_{1}}\right)^{2}+\frac{h \pi\left(t_{2}-t_{1}\right) \sin \left(\frac{\pi t_{1}}{4 \beta_{1}}\right)}{2 \beta_{1}} & ; t_{2} \leq t<t_{3} \\
+\frac{h \beta_{3} \sin \left(\frac{\pi\left(t-t_{2}\right)}{2 \beta_{3}}\right)}{\beta_{1}} &
\end{array}\right.
$$

$$
\begin{gathered}
\dot{\theta}(t)= \begin{cases}\frac{\pi h}{2 \beta_{1}} \sin \left(\frac{\pi t}{2 \beta_{1}}\right) & ; 0 \leq t<t_{1} \\
\frac{\pi h}{2 \beta_{1}} \sin \left(\frac{\pi t_{1}}{2 \beta_{1}}\right) & ; t_{1} \leq t<t_{2} \\
\frac{\pi h}{2 \beta_{1}} \cos \left(\frac{\pi\left(t-t_{2}\right)}{2 \beta_{3}}\right) & ; t_{2} \leq t \leq t_{3}\end{cases} \\
\ddot{\theta}(t)= \begin{cases}\frac{h \pi^{2}}{4 \beta_{1}^{2}} \cos \left(\frac{\pi t}{2 \beta_{1}}\right) & ; 0 \leq t<t_{1} \\
0 & ; t_{1} \leq t<t_{2} \\
-\frac{h \pi^{2}}{4 \beta_{1} \beta_{3}} \sin \left(\frac{\pi\left(t-t_{2}\right)}{2 \beta_{3}}\right) & ; t_{2} \leq t \leq t_{3}\end{cases}
\end{gathered}
$$

Functions $\theta(t), \dot{\theta}(t)$, and $\ddot{\theta}(t)$ utilised to obtain the graphics illustrated in Fig. 4:

$$
\theta(t)= \begin{cases}2 h \sin \left(\frac{\pi t}{4 \beta_{1}}\right)^{2} & ; 0 \leq t<t_{1} \\ 2 h \sin \left(\frac{\pi t_{1}}{4 \beta_{1}}\right)^{2}+\frac{h \pi\left(t-t_{1}\right) \sin \left(\frac{\pi t_{1}}{2 \beta_{1}}\right)}{2 \beta_{1}} & ; t_{1} \leq t<t_{2} \\ \frac{2 h \sin \left(\frac{\pi t_{1}}{4 \beta_{1}}\right)^{2}+\frac{h \pi\left(t_{2}-t_{1}\right) \sin \left(\frac{\pi t_{1}}{2 \beta_{1}}\right)}{2 \beta_{1}}}{+h\left[3 \pi\left(t-t_{2}\right)+\beta_{3} \sin \left(\frac{\pi\left(t-t_{2}\right)}{\beta_{3}}\right)\right]} & ; t_{2} \leq t<t_{3} \\ \frac{8 \beta_{1}}{2} & \end{cases}
$$




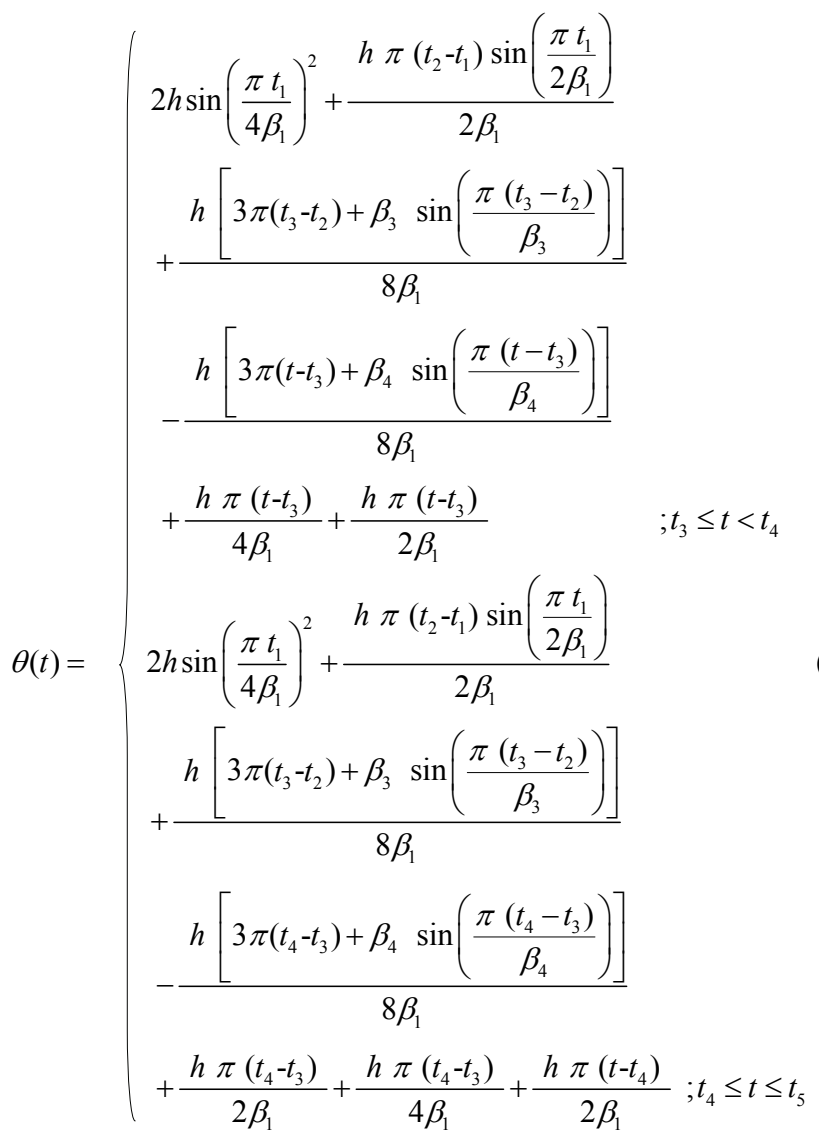

$$
\dot{\theta}(t)= \begin{cases}\frac{\pi h}{2 \beta_{1}} \sin \left(\frac{\pi t}{2 \beta_{1}}\right) & ; 0 \leq t<t_{1} \\ \frac{\pi h}{2 \beta_{1}} \sin \left(\frac{\pi t_{1}}{2 \beta_{1}}\right) t_{1} \leq t<t_{2} & ; t_{2} \leq t<t_{3} \\ \frac{\pi h}{4 \beta_{1}}\left[\frac{3}{2}+\frac{1}{2} \cos \left(\frac{\pi\left(t-t_{2}\right)}{\beta_{3}}\right)\right] & ; t_{4} \leq t<t_{5} \\ \frac{\pi h}{2 \beta_{1}}-\frac{\pi h}{4 \beta_{1}}\left[\frac{3}{2}+\frac{1}{2} \cos \left(\frac{\pi\left(t-t_{3}\right)}{\beta_{4}}\right)\right]+\frac{1}{2} \frac{\pi h}{2 \beta_{1}} & ; t_{3} \leq t<t_{4} \\ \frac{\pi h}{2 \beta_{1}} & ; 0 \leq t<t_{1} \\ \frac{h \pi^{2}}{4 \beta_{1}^{2}} \cos \left(\frac{\pi t}{2 \beta_{1}}\right) & ; t_{1} \leq t<t_{2} \\ 0 & ; t_{2} \leq t<t_{3} \\ -\frac{h \pi^{2}}{8 \beta_{1} \beta_{3}} \sin \left(\frac{\pi\left(t-t_{2}\right)}{\beta_{3}}\right) & ; t_{3} \leq t<t_{4} \\ \frac{h \pi^{2}}{8 \beta_{1} \beta_{4}} \sin \left(\frac{\pi\left(t-t_{3}\right)}{\beta_{4}}\right) & ; t_{4} \leq t<t_{5} \\ 0 & \end{cases}
$$

